\title{
The Application of Embedded Linux System in GPS Trade Terminal
}

\section{Device}

\author{
YanHua Zhou and YongChong Wu \\ JiangXi University of Technology
}

\section{Keywords: Embedded Linux System; GPS Trade Terminal; System operate}

\begin{abstract}
Embedded system has widely been permeated in many aspects, including scientific research, engineering design, military technology, various industrial and commercial cultures and arts, entertainment industry and people's daily life. With further development and promotion of foreign and domestic embedded products such as auto PC and set top box, embedded technology becomes more and more closely integrated with people's life. This paper explains the development of embedded Linux system in GPS grade terminal device and discusses several key technologies.
\end{abstract}

\section{Introduction}

With the rapid development of digital information technology and network technology in the PC (Post-PC) era, embedded system has widely permeated into aspects including scientific research, engineering design, military technology, various industrial and commercial cultures and arts, entertainment industry and people's daily life. With further development and promotion of foreign and domestic embedded products such as auto PC and set top box, embedded technology becomes more and more closely integrated with people's life.

Embedded operating systems began to be sued in various kinds of businesses since 1980s. Most of these operating systems are developed for proprietary systems, thus the current situation is that multiple forms of commercial embedded operating systems contend against each other. In the future society, there will be more and more circumstances where embedded systems are used. People can stay away from computer, but they cannot be separated from embedded system. Embedded system may exist in every corner of people's life. All the household appliances are managed and connection between family and external network is controlled by a center, which is controlled by embedded system. It makes people's life more convenient. If the doorknob of the hotel is installed with electronic identification control system, the door will open automatically according to the hands of visitors with the help of embedded system.. What's more, auto PC is able to judge the specific position through GPS (Global Positioning System) and determine which way is more convenient by embedded intelligence system. In addition, embedded system can be installed in equipment and instruments of the hospital to improve the success rate of surgery and decrease the pain of patients by choosing and controlling instruments.

Commercial embedded systems and proprietary operating systems developed since 1980s, which has more than 20 years of experience. Currently, the range of the application of embedded systems is extensive. Some famous embedded systems are introduced as follows. 
Last year, VxWorks of WindRiver Company merged pSOS of ISI Company, making this company now have two RTOS systems. VxWorks is the most widely used system with the highest market occupancy in embedded system field. It supports multiple processors, including x86, i960, Sun Sparc, Motorola MC68xxx, MIPS RX000 and POWER PC, etc. It applies the environment which is incompatible for UNIX and adopts GNU compiling and debugger. And most VxWorks API are proprietary.

Development of embedded systems of China also has made certain achievements, including EEOS of Chinese Academy of Sciences, HOPEN OS of Hopen and so on. However, Chinese products are auxiliaries and extensions of foreign companies to a great extent. Domestic products have low technical content.

In recent years, due to treating freedom, free of charge and open source code, Linux suddenly rises as a new force in the operating system market through efforts of programmers from the Internet and all over the world and the support from computer giants such as IBM and SUN. Server edition Linux and desktop edition Linux and embedded Linux have been extensively put into application. Application and application development based on Linux have permeated into various fields. Due to advantages such as low software licensing cost, abundant application development talent resources and convenience for developing individual and industry application, Linux is developing with steady pace in embedded development field, and now Linux is widely used in various embedded applications, including cell phone, internet device, thin client, firewall, industrial robot and basic equipment, such as mobile phone.

PDA refers to personal digital assistant and a digital tool to assist personal work just as its name implies. In fact, PDA should be divided into four categories, electronic dictionary, palm computer, hand-held computer device and personal communication assistant machine, while the last two have been slowly integrated together because of the development of technology and market.

GPS is a navigation positioning, timing and velocity-measuring system with globality, omnipotency (land, ocean, aviation and spaceflight) and all-weather advantages developed by US army at the beginning of 1970s on the basis of "meridian transit satellite navigation positioning. GPS is composed of three sub-systems, which includes space satellite system, ground monitoring system and user receiving system.

This paper introduces a GPS trade mobile terminal program, which can be simply described as GPS + PDA + mobile phone on function. In other words, GPS trade terminal is a GPS receiver, a PDA and a mobile phone as well. This system includes a set of complete GPS user receiving system on GPS function, namely, it includes GPS satellite data receiving module on hardware and GPS data processing software on software. Apart from GPS function, this program also includes functions of PDA and mobile phone. It can be said that GPS trade terminal of this program is a SmartPhone product with GPS function.

\section{Method}

The whole system is composed of following several parts: MC9328MX1 processor, GSM/GPRS wireless module, GPS module, memorizer module, display module, system reset module, memory expansion module, PC synchronous interface module, audio decoding module, key, system power supply module, debugging interface module and digital camera interface. Fig. 1 shows the structure diagram of the whole system. 


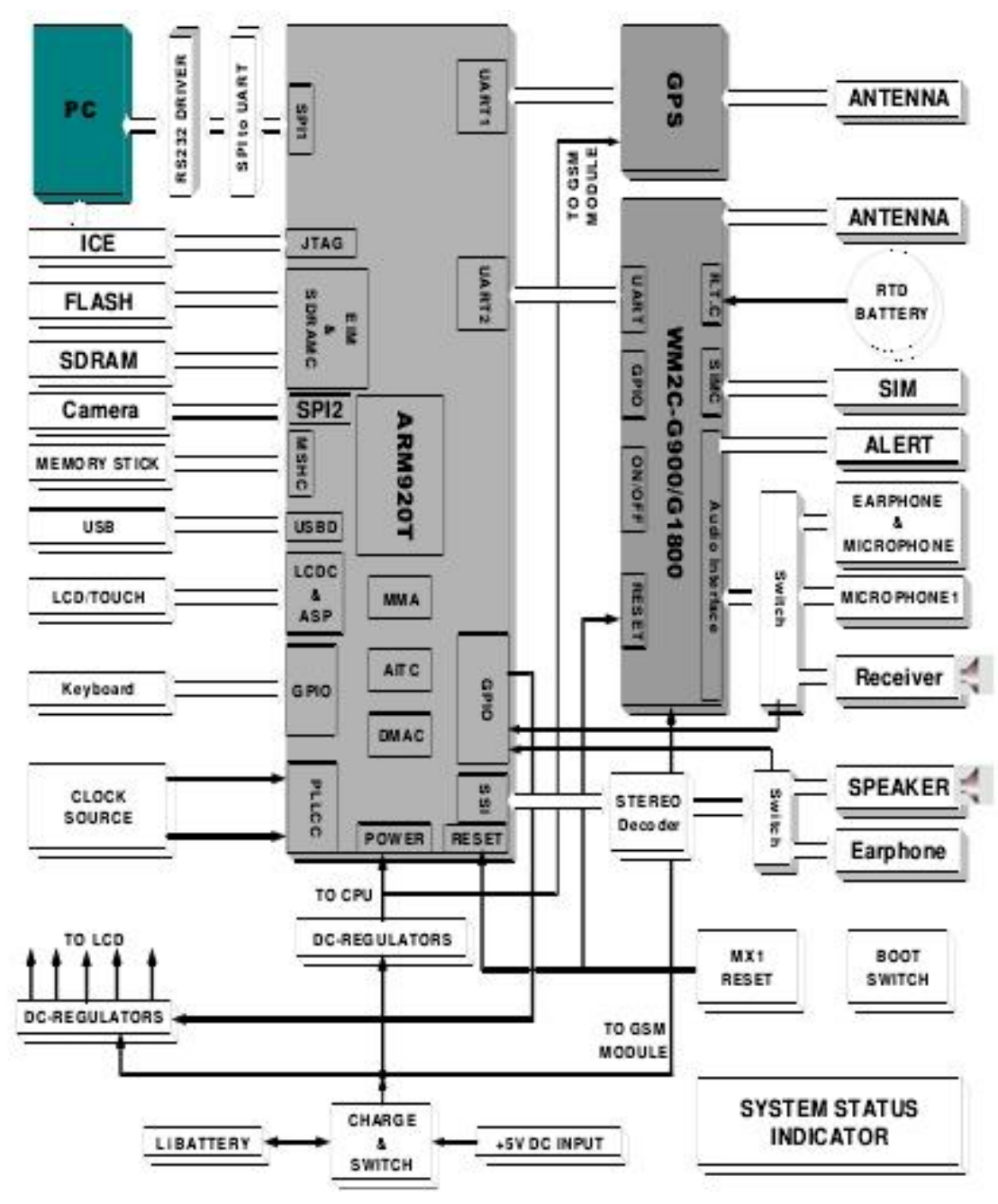

Fig. 1 System Diagram

Since Motorola MX1 processor is based on ARM framework, development environment based on ARM has to be constructed to carry out Linux embedded development. The so-called ARM cross compiling environment is to build a constructional environment to compile kernel and application program running in the hardware environment as shown in Table 1 on developing main engine. This set of ARM cross compiling environment is composed of a set of modules used for compiling, assembly, linking kernel and application program. These modules include:

Binutils - used for operating utility program collection of binary file, including utility programs such as ar, as, objdump and objcopy.

Gcc-GNU C compiler. Glibc-all users application programs are linked to C library (kernels without using any $\mathrm{C}$ library functions and other application programs can be compiled under the circumstance of absence of this library).

Debugging is a necessary link in the process of developing embedded system. Apparent difference exists between general common desktop operating system and embedded operating system under debugging environment. As for general common desktop operating system, routines of debugger and being debugged are often two processes operated on the same machine and the same operating system. Debugger process is to control and visit the process of being debugged through calling interface (ptrace debugging of early UNIX system and current process file system) specially offered by operating system. Embedded operating system which is also called remote debugging, is 
to offer flexible and convenient debug interface for system developers. Debugger is an application program running on general common desktop operating system. The program being debugged is running on embedded operating system (target operating system) based on specific hardware platform. It results in following problems: how debugger communicates with the program being debugged, how to timely inform debugger when the program being debugged has abnormality, how debugger controls and visits the program being debugged, how debugger identifies multitask information related to the program being debugged and controls certain specific task and how debugger processes some information related to target hardware platform (e.g. register information of target platform and disassembling of machine code). Generally speaking, all works of development of embedded application software by using integrated development environment, including editing, compiling, assembly and link, can be completed on PC machine. Debugging can only be completed through cooperating with other modules or products. Functions that debuggers need generally contain memory view, register browse, single step/ breakpoint setup, track, program download execution, Flash programming, operating system task-level state view, etc.

Kernel is the core of operating system. It is responsible for the process of management system, internal storage, and device driver, file and network system and determines performance and stability of the system. Since the source program of Linux is fully opened, everyone can modify the kernel and publish it for others' use as long as it follows GPL. In the design and application of embedded Linux, perhaps there is no need to frequently upgrade the kernel. However, for the target board to support more peripherals, design of the device driver according to users' needs is necessary, or sometimes some modules in kernel need to be redesigned. All these works including recompiling kernel make users be more familiar with the customized system. To accurately and rationally set the compiling and configuration option of the kernel, generally there are four things to be taken into account to obtain the code of function required by compiling system:

--Kernel of custom compilation runs faster (have fewer codes)

--The system will have more internal storage (the kernel won't be exchanged to virtual memory)

--Unwanted functions being compiled into kernel might increase loopholes utilized by system attacker

--The way of compiling certain function into module is slower than the one of compiling into kernel

Although the above-mentioned four points are for mature Linux suite such as Redhat Linux (run on X86 platform), it makes preparation for and is the only way for building embedded Linux operating system.

In the embedded system, significance and function of bootloader are similar to BIOS on PC. It initiates main parts on development board such as CPU, SDRAM, FLASH and serial port. Bootloader can be used to download files to development board, view category, burn flash and start system, etc. In fact, a bootloader with powerful functions is almost equivalent to a mini operating system.

1. Initiate the speed of basic hardware-CPU, memorizer sets timing, interrupts and detects the size of ram;

2. Initiation is required to read the device of Image;

3. Reserve a continuous physical memory for image, including detailed address and executive address of start;

4. Copy image to continuous address space; 
5. Use the amount of $\mathrm{r} 0=0, \mathrm{r} 1=$ machine structural system of MMU to call image and close Caches.

6. Initiate serial port and GPIO port and LED for debugging.

Special software can directly interact with flash device on remote system and install bootloader on given location of flash. Flash device is a special chip, whose function is similar to that of memory device, and it can store information durably, namely it won't erase its contents in reboot.

\section{Conclusions}

Based on implementation methods above, GPS trade terminal system developed based on embedded Linux is basically completed. Currently, various of device driver programs including boot load program, customized kernel and touch screen, root file system and GUI application functions such as GIS required by the program have been implemented based on the hardware platform of Motorola MX1. The system has inherited various kinds of outstanding characteristics of Linux, such as fewer resources occupied and high operating efficiency and being able to operate in a long-term and stable manner on hardware platform. For the next step of development, efforts shall be made in optimizing embedded Linux system. For instance, now the run-up time (from power on to the completion of GUI main interface start) of system is about 20 seconds, which is too long for a PDA mobile phone product. It requires to be optimized to reduce the run-up time, e.g. modifying the time occupied by operation in kernel such as unnecessary hardware test and initialization. The application of embedded Linux system in PDA device is not a new trial any more but an increasingly mature development selection. It is believed that there will be more types of Linux PDA and Linux mobile phone showing up in everyday lives in the future. The "wireless" spring of Linux system is approaching.

\section{References}

[1] Thorstensen B, Syversen T, Bjørnvold T A, et al. Electronic shepherd-a low-cost, low-bandwidth, wireless network system[C]//Proceedings of the 2 nd international conference on Mobile systems, applications, and services. ACM, 2004: 245-255.

[2] Chang Y F, Chen C S. Smart phone-the choice of client platform for mobile commerce[J]. Computer Standards \& Interfaces, 2005, 27(4): 329-336.

[3] Jobs M, Lantz F, Lewin B, et al. WBAN mass: A WBAN-based monitoring application system[J]. 2009.

[4] Nagata K, Yamaguchi S, Ogawa H. A power saving method with consideration of performance in android terminals[C]//Ubiquitous Intelligence \& Computing and 9th International Conference on Autonomic \& Trusted Computing (UIC/ATC), 2012 9th International Conference on. IEEE, 2012: 578-585.

[5] Yiqin L, Kanghua Y, Yuan L. An Implementation of Embedded Geographic Information System Based on Cloud Computing[C]//Circuits, Communications and System (PACCS), 2011 Third Pacific-Asia Conference on. IEEE, 2011: 1-4. 
[6] Zhifu W, Zhenhui R, Baozhong W. Development of the Electric Energy Collection System Based on Embedded LINUX[C]//Electronic Measurement and Instruments, 2007. ICEMI'07. 8th International Conference on. IEEE, 2007: 2-525-2-528.

[7] XI L, CHE Y, JI Y, et al. Research on data collection of production area of agricultural product based on embedded system [J][J]. Journal of Henan Agricultural University, 2009, 1:020.

[8] Bakker J D, Langendoen K, Sips H. LART: Flexible, low-power building blocks for wearable computers[C]//Distributed Computing Systems Workshop, 2001 International Conference on. IEEE, 2001: 255-259.

[9] Rennie C J, Groeneweg K K. Smart modem device for vehicular and roadside applications: U.S. Patent 7,355,509[P]. 2008-4-8.

[10] Tzamaloukas A. Enhanced mobile communication device with extended radio, and applications: U.S. Patent 6,925,378[P]. 2005-8-2. 